Rev. Elev. Méd. vét. Pays trop., 1973, 26 (4) : 99 a - 111 a

\title{
La commercialisation de la viande bovine au Sénégal
}

\author{
par M. S. DiAllo (*), M. BA (**), A. N'DAO (***) \\ et A. L. N'DIAYE $(* * * *)$
}

\begin{abstract}
RESUME
Ce document décrit les différents stades actuels de la commercialisation de la viande au Sénégal.

Après en avoir analysé les insuffisances à tous les niveaux, il propose les améliorations à entreprendre et la fixation de nouveaux prix de la viande.

Des statistiques concernant l'évolution des abattages et celle de la consommation de viande bovine sont jointes en annexe.
\end{abstract}

\section{INTRODUCTION}

L'approvisionnement en viande des populations pose des problèmes de plus en plus aigus et difficiles à résoudre et la conjoncture, à l'échelon mondial, est une pénurie grave. En fait, on ne voit même pas de façon claire, malgré les recherches conduites ça et là pour la mise au point de produits de substitution, comment combler le déficit dans un avenir prévisible.

Cependant, après les cris d'alarme de la FAO et de l'OCAM, la sécheresse exceptionnelle de l'hivernage 1972 précédée par 4 années successives de déficit pluviométrique dans l'ensemble de la zone sahélo-soudanienne d'Afrique, on a pu noter une prise de conscience de plus en plus nette de la part des responsables de différents pays et le Sénégal, encore importateur net de viande malgré un cheptel bovin important, accorde désormais une haute priorité au développement de ses ressources animales.

Le troupeau bovin sénégalais était estimé à environ 2670000 têtes à la fin 1971 et avait fourni quelque 29300 tonnes de viande carcasse et 7300 tonnes d'abats, soit un chiffre d'affaires au niveau de la boucherie de 4 milliards de francs CFA. Dans ce total, l'agglomération dakaroise intervenait respectivement pour 9285 et 2300 tonnes.

Les abattages contrôlés de bovins ont porté sur 140722 têtes correspondant à 19429 tonnes de viandes (voir tableau I).

La production et l'exploitation du bétail sont encore, pour l'essentiel, du type extensif traditionnel basé sur la vaine pâture. Toutefois, certaines régions du bassin arachidier pratiquent couramment l'embouche paysanne alors que l'embouche intensive industrielle s'est implantée et se développe dans le Cap-Vert depuis 3 ans.

(*) Direction de l'Elevage et des Industries Animales, Dakar.

(**) Société d'Exploitation des Ressources Animales du Sénégal (SERAS), Dakar.

(***) Directeur de l'abattoir de Dakar.

(***) Ecole inter-Etats des Sciences et Médecine vétérinaires, Dakar. 
TABLEAJ N $\mathrm{N}^{\bullet} \mathrm{I}$

Evolution des abattages de bovins au Sênëgal entre 1961 et 1971 .

\begin{tabular}{|l|c|c|c|c|c|c|}
\hline \multirow{2}{*}{ Annêe } & \multicolumn{3}{|c|}{ Abattage s contrôlës } & \multicolumn{3}{c|}{ Abattages estimês } \\
\cline { 2 - 7 } & $\begin{array}{c}\text { Nombre } \\
\text { de têtes }\end{array}$ & $\begin{array}{c}\text { Poids en } t \\
\text { carcasses }\end{array}$ & $\begin{array}{c}\text { Poids en } t \\
\text { abats }\end{array}$ & $\begin{array}{c}\text { Nombre } \\
\text { de têtes }\end{array}$ & $\begin{array}{c}\text { Poids en } t \\
\text { carcasses }\end{array}$ & $\begin{array}{c}\text { Poids en } t \\
\text { abats }\end{array}$ \\
\hline 1961 & 109369 & 14059 & 3515 & 157747 & 19705 & 4926 \\
1962 & 110127 & 13671 & 3418 & 161943 & 20011 & 5003 \\
1963 & 116872 & 14322 & 3580 & 170140 & 22118 & 5529 \\
1964 & 116080 & 14371 & 3593 & 181757 & 22183 & 5546 \\
1965 & 115974 & 15074 & 3768 & 181580 & 23258 & 5714 \\
1966 & 121666 & 15817 & 3954 & 199500 & 25935 & 6484 \\
1967 & 139706 & 18708 & 4677 & 201100 & 26401 & 6600 \\
1968 & 150730 & 20181 & 5045 & 223150 & 29210 & 7302 \\
1969 & 145861 & 19456 & 4864 & 220550 & 29492 & 7373 \\
1970 & 142563 & 19698 & 4924 & 224000 & 30300 & 7575 \\
1971 & 140722 & 19429 & 4857 & 217000 & 29310 & 7327 \\
\hline
\end{tabular}

NB Le poids des abats est estimé à 25 p.100 du poids de 1a carcasae.

TABLEAU N*II

Evolution des abattages contrôlês de bovins à Dakar et banlieue entre 1961 et 1971 .

\begin{tabular}{|c|c|c|c|c|c|c|c|c|c|}
\hline \multirow[b]{2}{*}{ Année } & \multicolumn{3}{|c|}{ Abat toir de Dakar } & \multicolumn{3}{|c|}{ B a n 1 i e ue } & \multicolumn{3}{|c|}{ T o t a u $x$} \\
\hline & $\begin{array}{l}\text { Nombre } \\
\text { de têtes }\end{array}$ & $\begin{array}{l}\text { Poids en } t \\
\text { carcasses }\end{array}$ & $\begin{array}{c}\text { Poids en } t \\
\text { abats }\end{array}$ & $\begin{array}{l}\text { Nombre } \\
\text { de têtes }\end{array}$ & $\begin{array}{l}\text { Poids en } t \\
\text { carcasses }\end{array}$ & $\begin{array}{c}\text { Poids en } t \\
\text { abats }\end{array}$ & $\begin{array}{l}\text { Nombre } \\
\text { de têtes }\end{array}$ & $\begin{array}{c}\text { Carcasses } \\
\text { en } t\end{array}$ & $\begin{array}{l}\text { Abats } \\
\text { en } t\end{array}$ \\
\hline 1961 & 42684 & 5549,0 & 1387,2 & 1670 & 217,1 & 54,2 & 44354 & 5766,1 & 1441,4 \\
\hline 1962 & 37833 & 4565,9 & 1142,5 & 3757 & 450,7 & 112,6 & 41590 & 5016,6 & 1254,1 \\
\hline 1963 & 41434 & 4888,9 & 1222,2 & 4690 & 519,3 & 129,8 & $46 \quad 124$ & 5408,2 & 1352,0 \\
\hline 1964 & 38495 & 4279,9 & 1069,9 & 4415 & 235,5 & 152,8 & 42910 & 4515,4 & 1222,7 \\
\hline 1965 & 39727 & 4914,5 & I 228,6 & $487 B$ & 585,3 & 146,3 & 44605 & 5499,8 & 1374,9 \\
\hline 1966 & 44729 & 6651,4 & I 662,8 & 5171 & 334,6 & 83,6 & 49900 & 6986,0 & 1746,4 \\
\hline 1967 & 48504 & 7445,6 & 1861,4 & 5438 & 429,9 & 107,4 & 53942 & 7875,5 & 1968,8 \\
\hline 1968 & 49417 & 7578,9 & 1894,7 & 6167 & 647,5 & 161,8 & 55584 & 8226,4 & 2056,5 \\
\hline 1969 & 54488 & 8041,4 & 2010,3 & 6289 & 853,5 & 213,3 & 60777 & 8894,9 & 2223,6 \\
\hline 1970 & 56914 & 8745,8 & 2192,4 & 6718 & 799,0 & 149,7 & 63632 & 9544,8 & 2342,1 \\
\hline $197 I$ & $54 \quad 427$ & 8091,5 & 2022,0 & 7474 & 1193,6 & 298,4 & 61901 & 9285,1 & 2320 \\
\hline
\end{tabular}

NB Le poids des abats est estimé à 25 p.100 dư poids de la carcasse.

TABLEAU $N^{\bullet}$ III

Abattages contrôlés de bovins à l'abattoir de Dakar en 1971 : répartition par catégories d'animaux.

\begin{tabular}{|l|c|c|c|c|}
\hline Gatégories & $\begin{array}{c}\text { Nombre } \\
\text { de têtes }\end{array}$ & $\begin{array}{c}\text { Pourcentage } \\
\text { du total }\end{array}$ & $\begin{array}{c}\text { Poids en } t \\
\text { carcasses }\end{array}$ & $\begin{array}{c}\text { Pourcentage du } \\
\text { poids total }\end{array}$ \\
\hline Tauresux & 1266 & 2,32 & 189900 & 2,34 \\
Boufss & 48991 & 90,03 & 7392960 & 91,36 \\
Vaches stériles & 2800 & 5,14 & 420000 & 5,19 \\
Veaux & 1370 & 2,51 & 88682 & 1,11 \\
\hline Totaux & 54427 & - & 8091542 & - \\
\hline
\end{tabular}


La commercialisation du bétail vivant comme la distribution des viandes connaissent encore des insuffisances graves. Les intermédiaires sont nombreux, l'accès au crédit bancaire quasi inexistant. Une note optimiste cependant, les décisions arrêtées par le Gouvernement à l'issue du Conseil interministériel du 21 décembre 1972 consacré à l'élevage.

Nous n'aborderons, dans la présente communication, que les problèmes posés par la commercialisation de la viande bovine à l'intérieur du Sénégal. En effet, les importations de viande sont négligeables et les exportations encore embryonnaires.

\section{L'APPROVISIONNEMENT DU SENEGAL EN BOVINS DE BOUCHERIE}

\section{Le marché de Dakar}

L'agglomération dakaroise dispose d'un foirail unique, celui de Thiaroye, situé à $15 \mathrm{~km}$ à l'est de la ville.

Les 60000 bovins que ce marché voit passer dans l'année sont presque exclusivement des zébus dont les 10 p. 100 sont d'origine mauritanienne. Le restant est représenté par le Gobra sénégalais. Les animaux arrivent à pied à travers la zone sylvo-pastorale et le bassin arachidier après plusieurs étapes, le long de circuits traditionnellement établis.

L'approvisionnement qui est à son optimum dès après la saison des pluies (novembredécembre), décroît progressivement à partir du mois d'avril pour devenir réellement difficile en saison des pluies (juin à septembre). Bien entendu les cours de la viande suivent dans le même temps une évolution inverse.

Il faut noter cependant quelques apports en bêtes de qualité provenant de l'embouche paysanne dans le Nord-Est du Bassin arachidier mais également de la ferme de Bambylor près de Dakar. Il s'agit d'animaux pesant entre 400 et $500 \mathrm{~kg}$ et dont le rendement en viande atteint ou dépasse 53 p. 100.

\section{Les marchés de l'intérieur}

Les principaux marchés de l'intérieur sont Louga, Dahra, M'Backé, Diourbel, Kaolack et Thiès. Thiès, noud stratégique, bénéficie d'une partie des convois dirigés sur la capitale. Kaolack est approvisionné à la fois par le Nord (zébus) et la route du Sénégal-Oriental (taurins N'Dama et métis Djakoré). La Casamance reste tributaire de l'Est du pays (N'Dama notamment). Tambacounda, lieu de convergence des pistes du Sud, voit passer beaucoup de «tiogals » mais demeure un centre de transactions moins important.

\section{LES AGENTS DE LA COMMERCIALISATION}

\section{Circuit vif}

On a coutume de qualifier d'archaïque le circuit commercial traditionnel. Les intermédiaires sont nombreux, les foirails ne sont, au mieux, que de simples enclos. Les animaux ne sont jamais pesés, les ventes se faisant à l'estime. D’une manière générale, le droit d'entrée au foirail municipal est de $100 \mathrm{~F}$ par bovin sur l'ensemble du territoire.

Il faut cependant reconnaître que compte tenu du mode actuel de production, de l'absence de crédit bancaire, ce circuit se révèle parfaitement adapté et très efficace.

\section{a) Le Dioula}

Il s'agit là, en fait, du véritable marchand de bétail, du détenteur de capitaux. Il parcourt la brousse d'un campement à l'autre pour collecter les animaux des éleveurs, soit par petits lots 
soit même à l'unité. De plus, il s'approvisionne directement sur les marchés des zones de production.

Les bovins ainsi achetés sont rarement payés comptant ou même intégralement au prix convenu. Les relations humaines interviennent pour beaucoup dans ce genre de transactions.

Les animaux acquis sont rassemblés en « tiogals » acheminés sur Dakar et les autres grandes villes.

\section{b) Le Téfanké}

Le champ d'action du téfanké est exclusivement le foirail. C'est lui l'intermédiaire quasi obligatoire entre acheteurs et propriétaires d'animaux et, de ce fait, perçoit une commission des deux côtés.

C'est l'agent qui possède le plus d'informations économiques, parfaitement au fait de la conjoncture et servi par une rare faconde, ce qui lui confère une stature particulière lors des transactions.

Si la vente se fait au comptant, le rôle du téfanké se réduit à faciliter l'opération en orientant les marchandages avec le plus grand tact.

Parfois, le téfanké agit en "marchand» en achetant fictivement les animaux qu'il revend comptant ou à crédit. Dans ce cas, il joue bien souvent le rôle de «logeur» du dioula ou de l'éleveur qui présente ses bêtes directement au foirail, le temps nécessaire à la récupération de la dette auprès de l'acheteur. Il peut alors tirer de son intervention une rémunération allant jusqu'à 5 p. 100 de la valeur du lot vendu.

\section{Circuit mort}

La profession de boucher reste encore très anarchique et pléthorique au Sénégal. Certains, notamment en brousse, exercent sans être patentếs alors que d'autres ne sont que des marginaux qui, bien qu'abattant irrégulièrement, contribuent néanmoins à l'encombrement d'une profession aux prises avec d'énormes difficultés. Rien qu'au niveau des chefs-lieux de régions et de départements, le nombre de bouchers recensés s'élève à 492 (voir tableau IV).

A côté des bouchers travaillent des tripiers plus ou moins spécialisés (abats rouges, abats blancs ou pieds de bœuf).

\section{a) A Dakar}

\section{Les bouchers}

Ils se différencient en plusieurs catégories suivant le volume de leurs affaires et l'échelon auquel se situent leurs activités.

- Chevillards

Ceux-ci se cantonnent au commerce de gros et de demi-gros (carcasses, demi-carcasses ou quartiers de bœufs). Cependant il sont parfois propriétaires, sur les marchés, d'un étal de vente au détail géré par leur représentant.

En fait plus de 63 p. 100 des abattages effectués à Dakar se trouvent concentrés entre les mains d'une vingtaine de chevillards. Durant l'exercice 1968-1969, ceux-ci ont eu à leur actif 5044 tonnes de viande sur un total de 7987, soit un chiffre d'affaires de 756,6 millions.

L'élément mauritanien demeure important puisque, parmi les 20 plus importants chevillards cités, 10 sont de nationalité mauritanienne et ont réalisé pendant la même période 3189 tonnes correspondant à un chiffre d'affaires de 478350 millions. 
TABLEAU $\mathrm{N}^{\circ} \mathrm{IV}$

Nombre de bouchers recensês dans les principales villes du Sênégal en 1972

\begin{tabular}{|c|c|c|}
\hline Ré g i o n s & $V$ i 11 es & Nombre de bouchers \\
\hline $\begin{array}{l}\text { Cap Vert } \\
\text { Casamance } \\
\text { Diourbel } \\
\text { Fleuve } \\
\text { Sénégal oriental } \\
\text { Sine-Saloum } \\
\text { Thiès }\end{array}$ & $\begin{array}{l}\text { Municipalitë du } \\
\text { grand Dakar } \\
\text { Ziguinchor } \\
\text { Oussouye } \\
\text { Bignona } \\
\text { Sédhiou } \\
\text { Kolda } \\
\text { Vélingara } \\
\text { Diourbel } \\
\text { Bambey } \\
\text { M'Backé } \\
\text { Louga } \\
\text { Kêbémer } \\
\text { Linguère } \\
\text { Saint-Louis } \\
\text { Dagana } \\
\text { Podor } \\
\text { Matam } \\
\text { Tambacounda } \\
\text { Bakel } \\
\text { Kédougou } \\
\text { Kaolack } \\
\text { Fatick } \\
\text { Foundiougne } \\
\text { Niaro } \\
\text { Gosaas } \\
\text { Kaffrine } \\
\text { Thiès } \\
\text { M'Bour } \\
\text { Tivaouane }\end{array}$ & $\begin{array}{r}225 \\
8 \\
2 \\
3 \\
3 \\
13 \\
5 \\
9 \\
6 \\
18 \\
20 \\
8 \\
3 \\
25 \\
4 \\
3 \\
4 \\
10 \\
7 \\
11 \\
16 \\
6 \\
2 \\
8 \\
8 \\
11 \\
33 \\
12 \\
9\end{array}$ \\
\hline Total & & 492 \\
\hline
\end{tabular}

Les chevillards fournissent:

- aux bouchers-détaillants;

- aux bouchers du secteur dit moderne et aux super-marchés qui s'adressent à une clientèle aisée;

- aux collectivités administratives (écoles, hôpitaux, prisons, armée) qui consomment plus de 550 tonnes de viandes par an.

Ils exportent également des viandes ou plus souvent des animaux sur pied vers des pays africains voisins (Libéria, Ghana) mais de façon sporadique et en quantités assez faibles.

- Bouchers-détaillants abattants.

Cette catégorie de professionnels se subdivise en détaillants abattants et détaillants non

Les premiers, qui se passent des services du chevillard, achètent des animaux vivants qu'ils transforment en carcasses.

Les seconds sont constitués de bouchers africains et de bouchers européens qui n'achètent que des quartiers ou des demi-bœufs auprès des chevillards.

\section{Les tripiers}

Ils se distinguent en plusieurs catégories suivant le niveau auquel ils se situent dans le circuit commercial, mais également selon la nature même des abats qu'ils commercialisent.

C'est ainsi qu'il existe des tripiers grossistes qui traitent avec les chevillards et approvi- 
sionnent les détaillants. Ceux-ci, qui règlent comptant les produits qu'ils prennent, permettent aux bouchers-abattants de payer tous les matins leur taxe d'abattage.

Suivant la nature des abats commercialisés, on peut classer les tripiers en :

- tripiers spécialisés dans les abats rouges (foie, cœur, langue);

- tripiers spécialisés dans les abats blancs (panses et boyaux);

- tripiers marchands de pieds de bœufs;

- tripiers spécialisés dans les bas morceaux autres que les abats (bosse, queue de bœuf, museau, bajoue, etc.) et qui pratiquent dans le même temps le commerce des cornes.

b) Dans l'intérieur du pays

Dans les villes de l'intérieur, comme en brousse, on ne rencontre pratiquement que des bouchers-abattants. Pour le reste, les choses se passent à peu près comme à Dakar.

\section{LA TRANSFORMATION DU BOVIN ET LA PREPARATION DE LA VIANDE}

\section{A Dakar}

Le bétail de boucherie est convoyé à pied du foirail de Thiaroye à l'abattoir à raison de $50 \mathrm{~F}$ par bovin. Une taxe de stabulation de $20 \mathrm{~F}$ par bovin et par jour est payée à l'abattoir.

Le boucher fait transformer ses animaux par une équipe de tueurs ( 8 personnes) qu'il rémunère partie en espèces et partie en nature. C'est ainsi que par bovin il doit, en plus de $300 \mathrm{~F}$ versés en espèces, céder la part coutumière d'une valeur de 600 à $1000 \mathrm{~F}$ suivant la saison et qui comprend le diaphragme, une partie des boyaux, de la graisse mésentérique, un morceau de rate, de collier et de muscles pectoraux. Cette rémunération est récupérée par le chef tueur qui procède au partage entre ses hommes. On imagine aisément tous les abus auxquels peut conduire le prélèvement de la part coutumière.

La collecte et la préparation des abats sont réalisées par les ouvriers tripiers qui opèrent à la tâche pour le compte des marchands d'abats.

La taxe d'abattage quant à elle s'élève à $8,50 \mathrm{~F}$ le $\mathrm{kg}$ de carcasse et se décompose comme suit :

- 6,50 $\mathrm{F}$ de droit d'usage;

$-2 \quad F$ de redevance municipale.

Un ressuage de 24 heures dans le frigorifique de l'abattoir est obligatoire pour toutes les carcasses.

\section{Dans les villes de l'intérieur}

Les pratiques sont identiques à quelques variantes près. Seuls les tarifs diffèrent. La taxe d'abattage par exemple n'est pas calculée au $\mathrm{kg}$ de carcasse mais à l'unité : $100 \mathrm{~F}$ par bovin en règle générale.

\section{LA DISTRIBUTION DES VIANDES}

\section{A Dakar}

Le transport des carcasses de l'abattoir au marché est assuré gratuitement par la Société d'Exploitation des Ressources Animales du Sénégal (SERAS) qui gère l'abattoir municipal. 
A défaut du camion isotherme de l'abattoir, le boucher paie au privé $200 \mathrm{~F}$ par bovin pour la même prestation de service.

Les viandes foraines introduites dans la commune de Dakar sont frappées d'une taxe de $10 \mathrm{~F}$ le $\mathrm{kg}$ versée au concessionnaire de l'abattoir municipal.

L'abattoir perçoit un droit d'entreposage dans ses chambres froides de $400 \mathrm{~F}$ par tonne indivisible et par jour, ou un forfait de $1000 \mathrm{~F}$ le $\mathrm{m}^{2}$ par quinzaine indivisible pour la location de case. De plus, chaque tonne supporte un droit de timbre de $5 \mathrm{~F}$.

S'agissant de la distribution, nous n'aborderons ici que le niveau du détail, les ventes de gros s'effectuant à l'abattoir de Dakar et, à une échelle beaucoup plus réduite, au marché de Sandaga.

\section{a) Les lieux de distribution}

\section{Secteur moderne}

Il s'agit de boucheries de type européen installées soit au marché Kermel, soit dans des supermarchés (Ranch Filfili, Printania, SAHM, Supermarché) soit encore dans des locaux spéciaux éparpillés à travers la ville.

On compte à l'heure actuelle une dizaine de boucheries modernes à Dakar, la plupart équipées en chambres froides.

\section{Secteur traditionnel}

Il concerne des bouchers plus ou moins importants, disposant de stalles dans les marchés ou possédant leurs installations propres.

D'une manière générale, les étals sont peu fonctionnels et l'hygiène laisse souvent à désirer.

\section{b) Les modes de distribution}

\section{Secteur moderne}

Ce secteur ne présente rien de particulier par rapport à ce qui se fait dans les pays européens. Les bouchers achètent la viande aux grossistes sous forme de pan-traité correspondant au quartier arrière prolongé jusqu'à la troisième côte et pesant en moyenne $54 \mathrm{~kg}$.

Les viandes sont découpées, désossées et parées par des garçons-bouchers qui récupèrent les os qu'ils vendent aux gargotiers. La graisse et les déchets de parage sont rétrocédés aux bouchers du secteur traditionnel.

\section{Secteur traditionnel}

Suivant son importance, le boucher prépare et vend directement ses viandes ou utilise les services d'un ou plusieurs aides rémunérés partie en nature et partie en espèces. Quand le boucher en titre se sert de vendeurs, il passe tous les soirs récupérer la recette.

La vente se fait au poids mais sous forme de viande avec os ne nécessitant donc ni parage, ni désossage. Parfois des morceaux de première catégorie sont désossés et vendus séparément, à la demande, à une clientèle spéciale.

Ne disposant pas de chambre froide, le boucher est le plus souvent obligé, en fin de journée, de céder à vil prix les invendus.

\section{La distribution des abats}

Elle est effectuée par les tripiers détaillants à l'abattoir même où se rendent certains consommateurs ou alors sur les marchés. La vente se fait exclusivement au tas, celui-ci comprenant à la fois des tripes, du gras, des déchets et de la " petite viande». 
c) Les cồts de la distribution et les prix

1. Secteur moderne

D'après les documents en possession de la Direction des impôts, les frais généraux d'une boucherie moderne représentent 22 p. 100 du prix d'achat des viandes.

Le quartier arrière "pan traité coupe pistolet» est acheté à $240-260 \mathrm{~F}$ le $\mathrm{kg}$ et les prix maximaux de vente pratiqués sont:

\begin{tabular}{|c|c|c|}
\hline Filet nature sans bavette & . & $836 \mathrm{~F} \mathrm{le} \mathrm{kg}$ \\
\hline Aloyau - faux filet - entrecôte - & rumsteak nature & $646 \mathrm{~F} \mathrm{le} \mathrm{kg}$ \\
\hline Filet nature avec bavette nature & $. \quad . \quad . \quad$. & $570 \mathrm{~F}$ \\
\hline Tranche nature. & . & $570 \mathrm{~F}$ \\
\hline Plat de côte - jarret . & 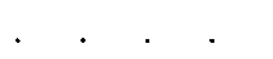 & 228 \\
\hline
\end{tabular}

2. Secteur traditionnel

Le boucher moyen s'approvisionne en avants qu'il paie 110 à $130 \mathrm{~F}$ le $\mathrm{kg}$ et qu'il revend entre 150 et $200 \mathrm{~F}$ suivant la saison bien que le prix officiel de la viande avec os soit de $200 \mathrm{~F}$.

Le coût de la distribution n'excède guère 10 p. 100 du prix d'achat de la viande par le boucher. La patente annuelle se situe autour de $5000 \mathrm{~F}$.

S'agissant des abats qui sont vendus au tas, le prix d'achat de gros se situe autour de $65 \mathrm{~F}$ le $\mathrm{kg}$ alors que le prix de vente varie entre 80 et $90 \mathrm{~F}$.

\section{Dans les villes de l'intérieur}

On peut affirmer que seul le mode traditionnel de distribution existe dans les villes de l'intérieur. Conformément aux habitudes culinaires, la viande est simplement débitée et vendue au $\mathrm{kg}$. Elle n'est ni désossée, ni parée. Le boucher est en général un abattant-détaillant.

Les abats sont vendus soit au tas, soit en même temps que la viande rouge, le boucher s'en servant alors pour faire « tomber» le plateau de sa balance.

\section{LA CONSOMMATION}

Le Sénégalais moyen est gros consommateur de poisson au détriment de la viande. Toutefois, l'élasticité de cette denrée par rapport au revenu est assez élevée car, dès que le niveau de vie augmente, la consommation familiale en viande crô̂t rapidement. Elle est supérieure à 1 au moins jusqu'à un niveau de revenu supérieur à deux fois le niveau moyen constaté. C'est ce qui ressort des études de budgets de ménages menées par le Service de la Statistique.

Le tableau $\mathrm{V}$ donne l'évolution de la consommation individuelle en viande et abats bovins pour Dakar et l'ensemble du pays de 1961 à 1971. Cette consommation se situe actuellement à $16 \mathrm{~kg}$ par habitant pour l'agglomération dakaroise et $9,5 \mathrm{~kg}$ pour l'ensemble du Sénégal si l'on ne tient pas compte des importations et exportations relativement négligeables de viande bovine morte.

\section{ACTIONS PROJETEES}

Les actions projetées en vue d'améliorer le circuit de commercialisation de la viande se situent à la fois au niveau du commerce du bétail sur pied, de la transformation et de la distribution des viandes. 
TABLEAU $\mathrm{N}^{\circ} \mathrm{V}$

Consommation individuelle de viande et d'abats bovins dans l'agglomération dakaroise et dans l'ensemble du territoire sénégalais de 1961 à 1971.

\begin{tabular}{|c|c|c|c|c|c|c|}
\hline \multirow{2}{*}{ Année } & \multicolumn{3}{|c|}{$D a k a r$ et b a.nlieue } & \multicolumn{3}{|c|}{$S$ é n é $g$ a 1} \\
\hline & $\begin{array}{l}\text { Population } \\
\text { humaine }\end{array}$ & $\begin{array}{c}\text { Viande }+ \text { abats } \\
\text { en } t\end{array}$ & $\begin{array}{c}\text { Consommation } \\
\text { individuelle } \\
\text { en } \mathrm{kg}\end{array}$ & $\begin{array}{l}\text { Population } \\
\text { humaine }\end{array}$ & $\begin{array}{c}\text { Viande }+ \text { abats } \\
\text { en } t\end{array}$ & $\begin{array}{c}\text { Consommation } \\
\text { individuelle } \\
\text { en } \mathrm{kg}\end{array}$ \\
\hline 1961 & 454100 & 7207,5 & 15,8 & 3142900 & 24631 & 7,8 \\
\hline 1962 & 498800 & 6270,8 & 12,5 & 3210400 & 25014 & 7,7 \\
\hline 1963 & 522800 & 6760,2 & 12,9 & 3275700 & 27647 & 8,4 \\
\hline 1964 & 548000 & 5738,1 & 10,4 & 3351100 & 27729 & 8,2 \\
\hline 1965 & 574300 & 6874,7 & 11,9 & 3388300 & 28972 & 8,5 \\
\hline 1966 & 575900 & 8732,4 & 15,1 & 3499500 & 32419 & 9,2 \\
\hline 1967 & 602000 & 9844,3 & 16.3 & 3577000 & 33001 & 9,2 \\
\hline 1968 & 630900 & 10282,9 & 16,2 & 3656300 & 36512 & 9,9 \\
\hline 1969 & 661300 & 11118,5 & 16,8 & 3738200 & 36865 & 9,8 \\
\hline 1970 & 693100 & 11886,9 & 17,1 & 3822500 & 37875 & 9.9 \\
\hline 1971 & $726 \quad 400$ & 11605,1 & 15,9 & 3908800 & $36 \quad 637$ & 9,3 \\
\hline
\end{tabular}

NB : La chute de la consommation constatêe pour Dakar entre 1962 et 1965 est ì imputer aux fraudes qui aṿaient alors cours à la pesée des carcasses à l'abattoir municipal.

-Il n'a pas êtê tenu compte des importations et exportations relativement négligeables de viande bovine morte.

\section{Le commerce du bovin sur pied}

La rationalisation et la moralisation du circuit vif tendront de plus en plus à mettre face à face le boucher et l'éleveur en supprimant la cascade d'intermédiaires qui s'intercalent encore entre eux.

Les dioulas et des téfankés seront progressivement reconvertis. En attendant, leur nombre sera réduit compte tenu des volumes des opérations sur chaque marché à bestiaux. Ceux qui seront autorisés à exercer après avis du Service de l'Elevage paieront patente et impôts sur les bénéfices industriels et commerciaux. Suivant le cas, un dioula pourra disposer d'un ou de plusieurs téfankés, ses employés, munis de cartes professionnelles à son nom. Ceux-ci joueront le rôle de commissionnaires en bétail.

L'accès au crédit bancaire est en principe acquis à la suite de la décision arrêtée à l'issue du Conseil interministériel du 21 décembre 1971 consacré à l'élevage. Les marchands de bestiaux, comme du reste les bouchers, relèveront désormais de la SONAGA (Société Nationale de Garantie), organisme institué par l'Etat pour assister les commerçants sénégalais. Ceci ne manquera pas de favoriser le paiement comptant et intégral des animaux d'autant que les coopératives d'éleveurs, elles, bénéficieront de prêts du nouveau Fonds mutuel de Développement rural (F.M.D.R.).

Par ailleurs, un effort sera fait pour la construction de foirails plus fonctionnels dans les principales villes de manière à faire adopter progressivement la vente des animaux au poids.

Dès lors, des cours du bétail sur pied pourront être imposés sur certains marchés et des mercuriales régulièrement diffusées à la radio pour l'information des diverses catégories professionnelles.

Les mesures nouvelles intéresseront d'abord le foirail de Dakar qui sera transféré dans la région de Thiès, à la limite de la zone déclarée indemne de maladies épizootiques qui s'étendra sur la totalité de la presqu'île du Cap-Vert. 
S'agissant du transport des animaux par voie ferrée, les études portant sur l'implantation de quais d'embarquement et de débarquement du bétail sont assez avancées. Ce mode de transport se révèle d'autant plus intéressant que la traversée de la zone agricole pour se rendre aux Centres d'abattage est très éprouvante pour les animaux (manque de pâturages sur le parcours) et est souvent source de conflits entre convoyeurs et cultivateurs.

Le transport par voie ferrée sera complété par un transport par camion-bétaillère.

\section{La transformation du bovin de boucherie}

A ce niveau, les améliorations portent à la fois sur la gestion des abattoirs régionaux et sur la réorganisation de la profession de boucher-abattant.

S'agissant des abattoirs, celui de Dakar servira de test. Un projet de réforme a déjà été proposé par la SERAS, Société gérante, à la municipalité, propriétaire de l'établissement.

La réforme proposée, qui n'est en fait qu'une mise en œuvre des clauses du cahier des charges de l'abattoir, consiste à n'admettre à l'usage des installations que du personnel appartenant à l'entreprise et à améliorer les conditions de travail par l'acquisition d'un équipement adapté. Le boucher n'aura plus qu'à confier son bovin à l'abattoir pour recevoir le lendemain la carcasse et le $5^{\mathrm{e}}$ quartier.

Les avantages seront multiples:

- contrôle efficace de la SERAS sur les tueurs-dépouilleurs d'où sauvegarde du matériel des abattoirs;

- amélioration de l'hygiène de l'abattage et de la préparation des viandes (tenues de travail);

-- revalorisation des cuirs par l'amélioration de la dépouille grâce à l'utilisation du perco;

- avantages sociaux et plus grande sécurité pour les tueurs-dépouilleurs (frais médicosociaux);

- suppression de la part coutumière, porte ouverte à des prélèvements abusifs sur les carcasses au grand dam des bouchers;

- augmentation des recettes de l'abattoir de 8 p. 100 environ;

- gain de $300 \mathrm{~F}$ environ par bovin abattu pour le boucher par rapport à la situation actuelle.

La réorganisation du métier de boucher passe par l'élimination des bouchers occasionnels, sans surface financière et qui ne font qu'encombrer une profession déjà confrontée à d'énormes difficultés.

Sur chaque marché, le nombre de bouchers sera limité au strict nécessaire. Seuls pourront exercer, les titulaires d'une licence délivrée après avis du Service de l'Elevage conformément aux dispositions de la loi $\mathbf{n}^{\circ}$ 71-47 du 28-7-1971 soumettant à autorisation ou déclaration préalable l'exercice de certaines professions industrielles, commerciales et artisanales et de son décret d'application $\mathbf{n}^{\circ}$ 71-1103 du 11-10-1971.

Par ailleurs, les nouveaux textes qui seront pris définiront de façon précise les attributions des d fférentes catégories de professionnels des métiers de la viande. Les chevillards se limiteront strictement au commerce de gros des viandes et abats.

\section{La distribution des viandes}

Nous nous situons ici au niveau du commerce de détail. Il reste entendu que dans les gros bourgs et les centres de brousse le bọucher pourrạ être à la fois abattant et détaillant.

Par contre, dans les grandes villes, ce commerce serait exclusivement réservé aux bouchers détaillants munis d'une licence et payant patente. Ils devront disposer d'un étal de vente public 
(marché) ou privé (boucherie) répondant aux normes d'hygiène requises. Ils s'approvisionneront en viandes et abats auprès des chevillards et certains maîtres-bouchers pourront se faire assister par des garçons-bouchers. Un projet, retenu au IV Plan de développement économique et social et assisté par le Canada, prévoit d'équiper les marchés urbains en chambres froides permettant ainsi le stockage des invendus.

\section{CONCLUSIONS}

Nous avons vu que la commercialisation des viandes bovines au Sénégal comporte pas mal d'insuffisances et ce à tous les niveaux (du producteur au consommateur), bien que la vocation pastorale du pays soit évidente.

Le circuit vif du bétail sur pied demande à être rationalisé et moralisé par l'élimination des intermédiaires non indispensables et la généralisation du règlement comptant grâce à l'accès au crédit bancaire des différentes catégories professionnelles (producteurs, marchands de bestiaux, chevillards). L'achat au poids mérite d'être imposé sur les principaux foirails.

Des modifications et des aménagements notamment en matière de gestion s'imposent au niveau des grands abattoirs.

Les améliorations du circuit mort doivent viser à réorganiser et désencombrer la profession de boucher.

En tout état de cause, l'application des mesures que nous préconisons ne manquera pas d'avoir un rôle stimulateur puissant dans le développement de l'élevage sénégalais.

\section{ANNEXE \\ Conclusions du Groupe de Travail chargé d'étudier l'approvisionnement régulier de Dakar en viande et la fixation des prix de cette denrée}

Le groupe de travail créé à l'initiative du Ministre du Développement rural et présidé par le Directeur de l'Elevage et des Industries animales était composé de représentants des Secteurs publics (Ministère du Développement rural et Ministère des Finances et des Affaires Economiques), semi-public (ranch de Doli, abattoir de Dakar) et privé (gérants de fermes d'embouche intensive et bouchers).

Le groupe, qui a déposé son rapport le 23 janvier 1973, a conclu à la nécessité d'une certaine hausse des prix et fait, à ce sujet, les propositions suivantes :

A. Propositions de prix au kg pour le bétail vif au foirail de Dakar :

1. Animal de $1^{\text {re }}$ qualité spécialement préparé pour la boucherie (rendement carcasse au moins égal à 50 p. 100):

- Fourchette de prix : $100 \mathrm{~F}$ à $120 \mathrm{~F}$ le $\mathrm{kg}$ vif, selon saison;

- Prix poindéré pour toute l'année : $110 \mathrm{~F}$ le $\mathrm{kg}$ vif.

2. Animal tout venant (rendement carcasse au moins égal à 45 p. 100):

- Fourchette de prix : $65 \mathrm{~F}$ à $75 \mathrm{~F}$ le $\mathrm{kg}$ vif selon saison;

- Prix pondéré pour toute l'année: $70 \mathrm{~F}$ le $\mathrm{kg}$ vif.

3. Animal maigre (rendement carcasse situé autour de 41 p. 100):

- Fourchette de prix : $50 \mathrm{~F}$ à $55 \mathrm{~F}$ le $\mathrm{kg}$ vif selon saison;

- Prix pondéré pour toute l'année : $52,50 \mathrm{~F}$ le $\mathrm{kg}$ vif.

B. Propositions de prix au kg pour la carcasse de bouf à la cheville aux abattoirs de Dakar :

1. Carcasse de première qualité :

- Fourchette de prix : $200 \mathrm{~F}$ à $230 \mathrm{~F}$ le $\mathrm{kg}$;

- Prix pondéré : $215 \mathrm{~F}$ le $\mathrm{kg}$. 
Le prix maximal de $230 \mathrm{~F} / \mathrm{kg}$ pour la carcasse de première qualité à la cheville implique les prix de vente maximaux suivants pour les quartiers correspondants, quel que soit le mode de découpe adopté :

- arrière $1^{\text {re }}$ qualité - prix maximal à la cheville: $275 \mathrm{~F} / \mathrm{kg}$;

— avant $1^{\text {re }}$ qualité - prix maximal à la cheville: $165 \mathrm{~F} / \mathrm{kg}$.

2. Carcasse animal «tout venant»:

- Fourchette de prix: $185 \mathrm{~F}$ à $205 \mathrm{~F}$ le kg;

- Prix pondéré pour toute l'année : $195 \mathrm{~F}$ le kg.

3. Carcasse animal maigre:

- Fourchette de prix: $150 \mathrm{~F}$ à $165 \mathrm{~F}$;

- Prix pondéré pour toute l'année : $157,50 \mathrm{~F}$.

\section{Prix de vente au $\mathrm{kg}$ au stade du détail :}

1. Dans les boucheries du secteur traditionnel, prix du $\mathrm{kg}$ de viande avec os:

Animal tout venant:

- Fourchette de prix : $230 \mathrm{~F}$ à $250 \mathrm{~F} / \mathrm{kg}$;

- Prix pondéré pour toute l'année: $240 \mathrm{~F} / \mathrm{kg}$;

Animal maigre:

- Fourchette de prix: $180 \mathrm{~F}$ à $200 \mathrm{~F} / \mathrm{kg}$;

— Prix pondéré pour toute l'année: $190 \mathrm{~F} / \mathrm{kg}$.

Les prix maximaux de vente de la viande avec os au détail dans le secteur traditionnel font ressortir un bénéfice brut sur prix de vente de l'ordre de $18 \%$ absolument indispensable pour couvrir frais généraux et pertes diverses et assurer une juste rémunération des professionnels de ce secteur.

2. Dans les boucheries du secteur moderne. Pour ce secteur, les calculs des prix de vente maximaux et valables toute l'année pour les différents morceaux ont été établis en fonction des critères ci-après:

-- Prix d'achat maximal de l'arrière de bouf de première qualité : $275 \mathrm{~F} / \mathrm{kg}$;

- Frais généraux sur prix d'achat: 18 p. 100;

- Bénéfice net sur prix de vente: 15 p. 100.

Le groupe de travail a arrêté ce pourcentage en fonction des considérations suivantes:

- Perte par ressuage égale à 7 p. 100 du poids de la carcasse au bout d'une semaine, délai normal de conservation de la viande dans les boucheries du secteur moderne;

- Emprunts bancaires effectués au taux de $11 \%$ par les bouchers; l'étal.

- Rétrocession à bas prix au secteur traditionhel des morceaux invendus après exposition d'une journée à

Compte tenu de ce qui précède, le groupe de travail propose le barème des prix de vente maximaux suivants pour les différents morceaux provenant de la découpe d'un arrière de bcuf de première qualité dans les boucheries du secteur moderne:

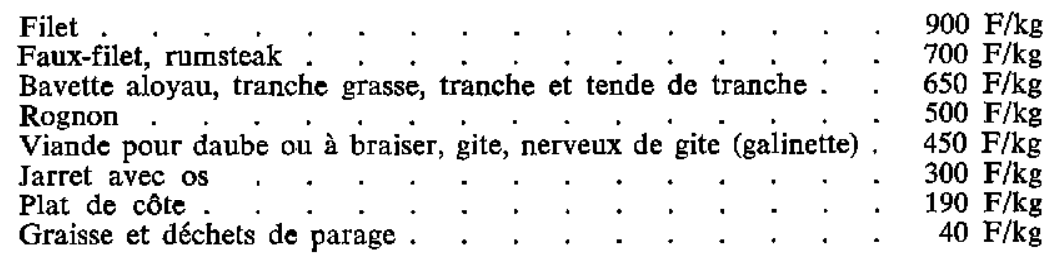

Le barème ci-dessus, appliqué à la découpe d'un arrière de bœuf effectuée le 7-12-1972, donné les résultats indiqués dans le tableau VI.

Prix achat maximal de l'arrière : $275 \times 58,500=16.087,50 \mathrm{~F}$.

Frais généraux $=\frac{16.087,50 \times 18}{100}=2.895,75 \# 2.896 \mathrm{~F}$.

Prix revient $=16.087,50+2.895,75=18.983,25$ \# $18.983 \mathrm{~F}$.

Prix vente selon « barème proposé » $=22.413 \mathrm{~F}$.

Bénéfice net $=22.413-18.983=3.430 \mathrm{~F}$.

$\% \mathrm{BN} /$ Prix de vente $=\frac{3.430 \times 100}{22.413}=15,30 \%$.

N.B.: Il convient de noter que des commissions régionales chargées de réviser les prix des viandes dans le sens d'une hausse ont été créées dans les différentes régions du Sénégal à l'initiative du Ministre des Finances et des Affaires économiques (cf. notes circulaires nos 8747 et 8748/DCI-P du 18-7-1972). 
TABLEAU $N^{\bullet} \mathrm{VI}$

\begin{tabular}{|c|c|c|c|c|}
\hline $\begin{array}{l}\text { Poids de } 1 \text { 'arrière : } 58,500 \mathrm{~kg} \\
\text { Découpe effectuée aprês } 24 \text { heures } \\
\text { de ressuage. }\end{array}$ & $\begin{array}{l}\text { Poids } \\
\text { en kg }\end{array}$ & $\begin{array}{l}\text { Pourcentage } \\
\text { des } \\
\text { morceaux }\end{array}$ & $\begin{array}{l}\text { Prix } \\
\text { maximaux } \\
\text { proposés }\end{array}$ & $\begin{array}{l}\text { Montant } \\
\text { en francs }\end{array}$ \\
\hline $\begin{array}{l}\text { Résultats : } \\
\text { a) Filet } \\
\text { b) Bavette d'aloyau } \\
\text { c) Tranche et tende de tranche } \\
\text { d) Faux filet } \\
\text { e) Tranche grasse } \\
\text { f) Rumsteak } \\
\text { g) Viande pour daube ou à braiser } \\
\text { h) Viande maigre pour hacher } \\
\text { (beefsteak) } \\
\text { i) Nerveux de gîte (galinette) } \\
\text { j) Gîte, gite à la naix } \\
\text { k) Plat de côtes } \\
\text { 1) Jarret avec os } \\
\text { m) Rognon } \\
\text { n) Graisse } \\
\text { o) Dêchets de parages) } 31,10 \text { p.100 } \\
\text { p) Os }\end{array}$ & $\begin{array}{l}1,250 \\
1,150 \\
4,200 \\
8,000 \\
3,400 \\
3,350 \\
3,500 \\
1,650 \\
1,500 \\
5,550 \\
4,000 \\
2,400 \\
0,350 \\
5,450 \\
5,250 \\
7,500\end{array}$ & $\begin{array}{r}2,13 \\
1,96 \\
7,17 \\
13,67 \\
5,81 \\
5,72 \\
5,98 \\
2,82 \\
2,56 \\
9,48 \\
6,83 \\
4,10 \\
0,59 \\
9,31 \\
8,97 \\
12,82\end{array}$ & $\begin{array}{l}900 \\
650 \\
650 \\
300 \\
650 \\
700 \\
450 \\
500 \\
450 \\
450 \\
190 \\
300 \\
500 \\
40 \\
40 \\
-\end{array}$ & $\begin{array}{cc}1 & 125,00 \\
747,50 \\
2 & 730,00 \\
5 & 600,00 \\
2 & 210,00 \\
2 & 345,00 \\
1 & 575,00 \\
& 825,00 \\
& 675,00 \\
2 & 497,50 \\
760,00 \\
720,00 \\
175,00 \\
218,00 \\
210,00 \\
-\end{array}$ \\
\hline Total & 58,500 & 99,92 & & $22 \quad 413$ \\
\hline
\end{tabular}

\section{SUMMARY}

\section{Beef commercialization in Senegal}

In this paper, the authors report the various current steps of beef commercialization in Senegal.

After a previous analysis of the deficiencies observed at all levels, they propose improvements to be undertaken and settlement of new prices for beef.

Statistics dealing with the evolution of slaughtering and of beef consumption are available in a joined enclosure.

\section{RESUMEN}

\section{Comercialización de la came bovina en Senegal}

Este trabajo describe los diferentes estadios actuales de la comercialización de la carne bovina en Senegal. Analiza todas las insuficiencias y propone mejoras que hay que emprender y la fijación de nuevos precios de la carne.

Un anejo da estadísticas concemiendo a la evolución de las matanzas y la del consumo de came bovina.

\section{BIBLIOGRAPHIE}

1. BA (M.) et N'DAO (A.). Note relative à la commercialisation et à l'organisation des marchés des viandes bovines dans la région dakaroise. Déc. 1969.

2. F.A.O. Commercialisation du bétail et de la viande en Afrique. Rome, F.A.O., 1960. (Coll. La Commercialisation, $n^{0} 3$ ).

3. LACROUTS (M.) et TYC (J.). Etude de l'équipement frigorifique du Sénégal. Situation et perspective en vue de l'amélioration de la production, de la transformation et de la distribution du poisson et de la viande. Deuxième partie: Les problèmes relatifs au bétail et à la viande. 1967 .

4. LEDUC (A. C.) et TYC (J.). Etude du marché de la viande dans l'agglomération dakaroise. 1963.

5. TROQUEREAU (P.J. A.). Les ressources animales du Sénégal. Leur exploitation. Perspectives d'avenir. Nov.-déc. 1960.

6. VALENZA (J.), DENIS (J. P.), DIALLO (M.S.) et THIONGANE (A.). La viande au Sénégal, production et hygiène. Perspectives. VII Journées médicales de Dakar, janvier 1971. 